\title{
Real-time Image Haze Removal Method for Fire Scene Images
}

\author{
Rui Xue $^{\mathrm{a}}$, Ming Zhong ${ }^{\mathrm{b}}$, Enxuan Zhang ${ }^{\mathrm{c}}$, Shasha Zhao ${ }^{\mathrm{d}, *}$, Dengyin Zhang ${ }^{\mathrm{e}, *}$ \\ ${ }^{1}$ College of Internet of Things, Nanjing University of Posts \& Telecommunications, Nanjing, WenYuan \\ Road, China \\ a 49063417@qq.com, b3 634334680@qq.com, c 87660665@qq.com, ${ }^{\mathrm{d}}$ zhaoss@njupt.edu.cn, e \\ zhangdy@njupt.edu.cn
}

Keywords: real time image haze removal, dark channel priori, guided filtering, histogram equalization

\begin{abstract}
A large amount of haze in the fire scene greatly affects the survey on the scene for fire fighters. The intelligent fire control can greatly improve the fire rescue efficiency and reduce the casualty rate. In this paper, an algorithm based on the hardware conditions of the intelligent fire helmet platform is proposed, which improves dark channel prior. Filtering partition and sampling are respectively used to quickly and accurately evaluate and repair the transmittance with a guided filter. Replacing the maximum value with an average one, the global atmospheric light is more efficiently and accurately estimated. The histogram equalization algorithm was finally carried out. The dehazing image contrast can be effectively improved using the histogram equalization algorithm. As a result, real-time and rapid fire scene image haze removal can be obtained at the same time, which will effectively help firefighters to spray treatment and finish on-site survey.
\end{abstract}

\section{Introduction}

Haze has a great impact on the image formation, especially for fire scene, where contains a large number of suspended particulate aerosol. It not only produces serious light diffuse reflection, but also absorbs a lot of light, resulting in greatly reduced visibility. As all the objects are blurred, the personnel investigation of fire rescue is seriously hampered.

Today, the image haze removing are mainly focused on outdoor haze images with a mature technology, where image enhancement and restoration were done by the physical model or priori information. [1, 2] There is little report about the development of the haze removing of the image taken in the fire scene, which is full filled aerosol. Additionally, compared with outdoor haze, the image of fire-scene has an inhomogeneous distribution including concentration and interfered degree. Fire scene images are more blurred, and the diffusion of the smoke has a certain direction. While, clear images are important and necessary for the firefighters to carry out fire rescue safely and efficiently. However, the existing algorithm of the image haze removal, including the image haze removal used in outdoor, cannot improve the image brightness and highlight the main objects in the image. As a result, a new way to haze removing for the images taken in the fire field with smoke characteristics is required.

In this work, based on the portability of rescue equipment, an improved dark channel prior algorithm is proposed, which leads to an implementation of real time image haze removal. The experimental results demonstrate the haze removed images can highlight the features of main objects on the rescue site, meeting the requirements of fire rescue.

\section{Experimental Procedure}

Based on the special fire environment and the characteristics of wearable devices, we make a research for image haze removal methods.

\subsection{Features of the Fire Scene}

Although the haze on the fire scene has the same points as the haze outside, there are many 
different characteristics because of the special limits of the fire [2].The fire scene usually have three kinds of phenomena including smoke, plume and smoldering. The smoke is a mixture of ash and gas, which flows through the heat by exchanging heat with air. After a period of time, convection also called Fire plume are formed with the heat action derived from the steady fuel combustion. Smoldering is formed from the solid combustion, which is a slow combustion without visible light [3].

The haze in the fire scene resulted from the three phenomena mentioned above is different from the mist that is in the natural environment within the following aspects [1] [4]:

1) The fog in the natural state is mainly liquid. Its transmittance is stronger than that of the smog. While the smoke in the fire field is mainly composed of solid particles and blocks light transmittance. As a result, fire scene images will be more blurred. Furthermore, the plume can give rise to a certain degree of light distortion.

2) Most of fog in the natural state is relatively slow. After a long time, the fog is evenly distributed. Meanwhile, the fog flow is largely uniform. However, in fire scene, the smoke begins to spread out from the burning point at the beginning of the fire. Moreover, a large amount of smoke will be rapidly produced from the combustion of the combustibles.

3) The flow of most of the haze in the fire scene is weaker than the other. The diffusion of the smoke has a certain direction.

At the same time, due to the fireman need carry a lot of equipment to enterer the fire field, and the algorithm run in most cases is also on the portable equipment. Therefore, the requirement of equipment operation ability is reduced under the premise of comprehensive consideration of the effect of dehazing.

\subsection{Real-time image haze removal method for fire scene images}

The existing image haze processing methods mainly include image enhancement and image restoration. The image enhancement can improve the contrast of the haze image, but some image information may be loosed. Haze image restoration is based on the conclusion of physical process with haze. Since it establishes physical model, it enhances the effectiveness of image processing. Meanwhile, it reduces image information loss, and makes up for the image distortion caused by haze interference. However, due to the darkness of the fire scene, there will be some black haze. The information in the dark will be loss in the image, affecting the subsequent image utilization. Therefore, dark channel prior treatment is included in our proposed algorithm. Based on the global atmospheric optical estimation, partitioning and sampling ways are carried out to improve the operation speed. The bootstrap filtering is done to repair the transmittance. Finally, histogram equalization algorithm is used to improve the whole image brightness.

The dark channel prior fog removal algorithm improves the estimation of the transmittance. According to the actual situation of the fire scene, it also improves the enhancement of the late image in the algorithm based on the image degradation model. The specific algorithm chart is shown in Figure 1.

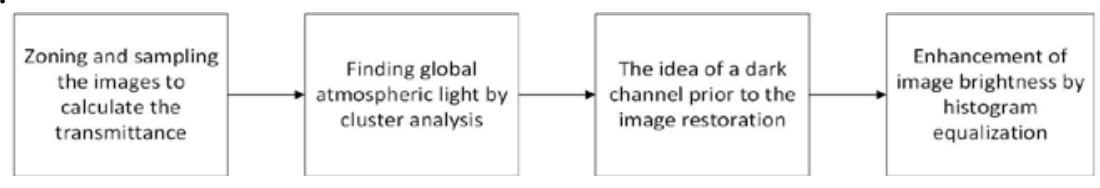

Figure 1 Improved dark channel prior algorithm flow chart.

The principle of dark channel prior can be expressed as [5]:

$$
J^{\text {dark }}(x)=\min _{y \in \Omega(x)}\left(\min _{c \in(r, g, b)} J^{c}(y)\right)
$$

where, $J^{\text {dark }}(x)$ is the colour image with three RGB channels and $\Omega(x)$ is a $n \times n$ size filter represented a minimum value of two times for a color image. The dark channel of the fog free image satisfies $J^{\text {dark }}(x) \rightarrow 0$.

The dark channel priori haze removal algorithm also combines the image degradation model [6]: 


$$
I(x)=J(x) t(x)+A(1-t(x))
$$

where, $I(x)$ is the fog image, $J(x)$ is the image after haze removal, $t(x)$ is the transmittance, and $A$ is the global atmospheric light.

The smart fire helmet is firstly sampled and partitioned. Then the transmittance is estimated based on the mean filter. Finally, the processed transmission map is used as the input graph. The reverse channel of the dark channel retains the depth of field information. It is used as a guiding graph to conduct the guided filter repair of transmittance. The guided filter repair can fine the transmittance. In order to improve the speed of calculation, the original image is sampled before the transmission rate is estimated.

\subsubsection{Subarea Estimation Method Based on Mean Filtering}

Firstly, the fog image is sampled. It is reduced to $1 / 4$ of the original image. According to the image degradation model, the value of the fog image will not be less than zero in general. [7] This will lead to the expression as

$$
I(x) \geq 0
$$

From (2), it can be obtained.

$$
J(x) t(x) \geq 0
$$

In combination with (2) and (4), the lower bounds of transmittance can be obtained.

$$
t(x) \geq 1-\frac{I^{c}(x)}{A_{0}}, c \in\{r, g, b\}
$$

The mean filter is performed on the right side of the equation and the equation (5) can be deduced as

$$
t(x) \geq 1-\frac{\underset{y \in \Omega(x)}{\operatorname{avg}\left(\min _{c \in\{r, g, b\}}\left(I^{c}(x)\right)\right.}}{A_{0}}
$$

Synthesis (5) and (6) can be obtained.

$$
t(x)=\max \left(1-\omega \frac{\underset{y \in \Omega(x)}{\operatorname{avg}\left(\min _{c \in\{r, g, b\}}\left(I^{c}(x)\right)\right.}}{A_{0}}, 1-\frac{\min _{c \in\{r, g, b\}}\left(I^{c}(x)\right.}{A_{0}}\right)
$$

To avoid the excessive fog and the loss of the depth of the whole image caused by the partial higher brightness after fixating $\omega, \omega$ is adjusted according to the brightness of the image.

$$
e(x)=\frac{A_{0}-\min _{c \in\{r, g, b\}}\left(I^{c}(x)\right)}{A_{0}}
$$

When $e(x)$ is relatively small, the estimation of the transmittance should be improved. So the estimated formula of $\omega$ is can be described as follows:

$$
\omega=\frac{l b\left(\left|\frac{A_{0}-\min _{c \in\{r, g, b\}}\left(I^{c}(x)\right) \mid}{A_{0}}\right|+1\right)}{K}+b, b \in(0,1), k>1
$$

where the value of $b$ is fixed, while the first half of the formula can be adjusted according to the brightness of the different image regions. By this adjustment, the image with high brightness can be hazy and the effect is more natural. 


\subsubsection{Guided Filter Repair}

In general, the concentrations of smoke in the fire scene are equivalent to the medium or large haze in the natural outdoor state. For heavy haze, the existed dark channel priors are not effective enough to do haze removing. Because the prior transcendental theory is not well precise and the edge is not good enough, the guided filter repair is carried out to fine the transmittance. The smart fire helmet takes the processed transmission picture as the input graph. A dark channel reverse diagram retains information on the depth of the field. The diagram is used as a guide graph. Then, the smart fire helmet conducts the guided filter repair the transmittance. Two equations are used to repair the transmittance:

$$
\begin{gathered}
t_{2}(x)=a_{k} F_{i}+b_{k}, \forall i \in \omega_{i} \\
t_{2}(x)=t(x)-n_{i}
\end{gathered}
$$

The formula (10) is used to repair the transmission rate to get the final transmittance, and the formula (11) is used to restrain the repair degree of the guide filter. Combined equation (10) and (11) as well as the minimization of the cost function, the expression of two parameters $a_{k}$ and $b_{k}$ can be obtained as

$$
\begin{gathered}
a_{k}=\frac{\frac{1}{|\omega|} \sum_{i \in \omega_{k}} F_{i} p_{i}-\mu_{k} p_{k}}{\sigma_{k}^{2}+\varepsilon} \\
b_{k}=p_{k}-a_{k} \mu_{k}
\end{gathered}
$$

where, $\mu_{k}$ is the average value of the boot graph in the local window, $\sigma_{k}$ indicates the variance of the boot graph in the local window, $\omega$ is the number of pixels in the local window, $p_{k}$ indicates the average value of the input graph in the local window and $\varepsilon$ is the custom parameter limiting the smoothness of the image is the custom parameter.

According to the general method of estimating the global atmospheric light $A[8]$, the primary dark colour image was firstly worked with the fog image. Then the first $0.1 \%$ pixels with the largest brightness are detracted and the maximum value of the corresponding pixel in the fog image is obtained which is as the value of A. However, there are four problems in this algorithm:

First, the sorting algorithm will be applied. Since only the maximum pixel value of the original image is used, it greatly reduces the efficiency of the image defogging.

Second, there is a white edge phenomenon due to the poor edge retention, resulting from the used fog transmittance obtained by the direct degradation model. However, in fact, there is no significant depth of field on the scene of the fire. A guided filtering method is adopted to repair the calculated transmittance of the image. As a result, the white edge phenomenon is avoided.

Third, according to this algorithm, the estimated value of atmospheric light is higher. After fog removal, the image will appear low brightness and gray concentration. It is not conducive to the investigation of the scene of the fire.

Fourth, the accuracy of atmospheric light estimation has an obvious effect on the results of image processing. As it is well known that, the hard estimate to the white objects within global atmospheric light will results in an in accurate estimated value when fog image is dark and colour distortion.

A highly efficient global atmospheric optical estimation method is used in the smart fire helmet. Cluster analysis also is used to find the atmosphere light tends to be white colour value. These favour us to calculate the colour value of atmospheric light and improve the processing efficiency of the fog image. In additional, it effectively avoids the distortion of the image colour. Thus, the brightness of $\mathrm{k}$ pixels is randomly selected as the initial center of mass. Then we calculate the distance from other pixels to these centers of mass. If point $p$ is closer to the nth center of mass, and 
point $\mathrm{p}$ belongs to class $\mathrm{n}$, the average value of pixel brightness will be calculated in the same class and as the new center of mass. The above procedure will be iterated until all the centroid does not change as well as the end of cluster analysis. The obtained maximum brightness value of the pixel in these points is taken as the value of global atmospheric light.

This method can effectively filter out the outliers and improves the efficiency of the calculation. For the image degradation, $J(x)$ of the image after bringing $A$ and $t(x)$ into the image degradation model is described as

$$
J(x)=\frac{I(x)-A}{\max \left(t(x), t_{0}\right)}+A, \quad t \geq t_{0},
$$
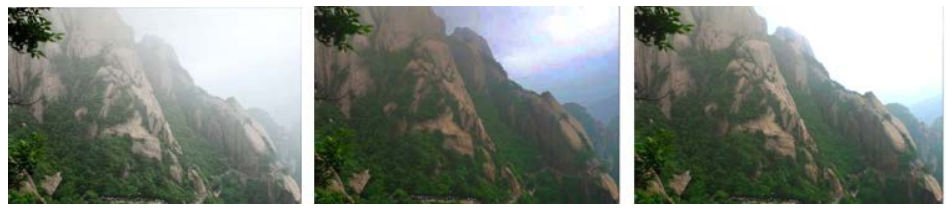

Figure 2 Image comparison of global atmospheric light from original map, non-cluster analysis and cluster analysis.

From the above chart, we can see that in the dark channel prior method, the selection of atmospheric light is prone to cause misjudgement. So, the selected atmospheric light is lighter than the real one in foggy image. The result of fog removal is obviously dim and even difficult to distinguish details [9]. The atmospheric luminance selected by cluster analysis is moderate, and the difference between the brightness and the original image is little.

\subsubsection{Improve The Brightness of The Image by Histogram Equalization Method}

When the atmospheric light estimation is used, the overestimation of the whole image will lead to a dark image. Due to the scene of the fire is poor and the image of fog is low the image after fog removal is dark and gray. Histogram equalization algorithm [10] is used to reduce the number of small gray pixel value, and compress dynamic range of them. Meanwhile it also can enhances gray pixel number of more value, and broaden their dynamic range. Finally, the distribution of fog image gray value distribution is more uniform. After the image histogram equalization processing, the contrast is improved and the image is clearer. As a result, we can selectively highlight the images associated with the rescue. So that it is convenient for rescue workers to quickly survey the scene of the fire.

\subsection{Results and Discussion}

\subsubsection{Results Comparison}

The heading of a section title must be 12-point bold, aligned to the left with a line space single and an additional space before of 18-point and after of 6- point. This paragraph should have first line hanging indent of 0.63 centimetres. The initial letters should be capitalized.

Dot should be included after the section title number. Software runs in smart fire helmet. And the top-down modular design is based on process oriented thinking. The functions are independent. Meanwhile, the final software products are reliable and expansible.

The fire scene images were carried out in the fogging experiment. So we can verify the effectiveness of the fire scene image processing and the efficiency of the processing. The images in the smoke environment are repeatedly tested and verified with the algorithm. It is proved that the operation speed of the image processing scheme is fast enough and the image defog is good enough. In the actual application, the investigation of the fire site can provide effective help.

However, as we all know, many foggy image restoration methods have been proposed and have made significant progress. In order to verify the advantages of our method, we conduct the experimental comparison with several state-of-the-art methods, including [7, 16]. We choose two challenging hazy images (a dense haze image of outdoor fire scene (Figure 3a) and a hazy image of indoor fire scene (Figure $3 f$ ) for the experimental comparison. The relevant comparison results are 
provided in Figures 3b-e and g-j. As shown in Figure 3, the images that are estimated using our method are more consistent with our intuition. It can easier find objects from the image using our method than those of $[14,15]$.

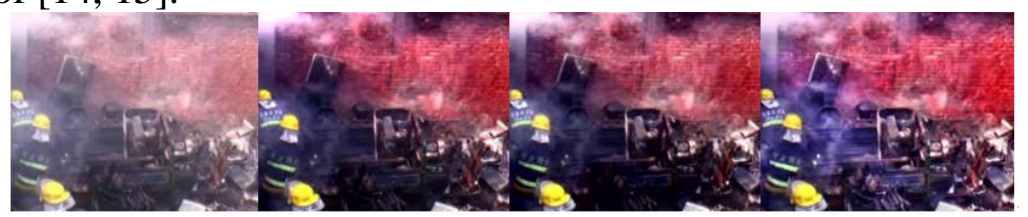

(a)

(b)

(c)

(d)

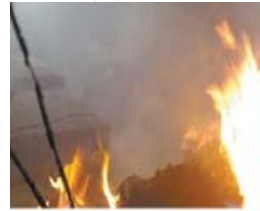

(e)

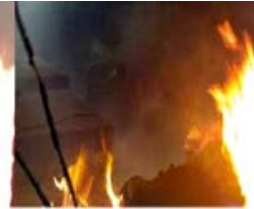

(f)

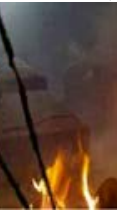

(g)

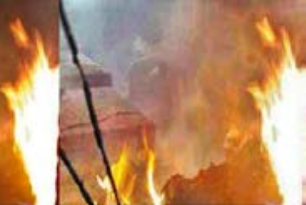

(h)

Figure 3 Comparison of the transmission maps that are estimated in the way reported within the way used in Ref.7, Ref.16, and this work. (a) A dense haze image of outdoor fire scene and (b-d) the corresponding images respectively recovered via Ref7, Ref.16 and our method.(e) A hazy image of indoor fire scene and (f-h) the corresponding respectively recovered via Ref.7 and Ref. 16 and our method.

\subsubsection{Qualitative Comparison}

In order to quantitatively evaluate and enhance visibility and dehazing effect, four employed indicators are widely used in terms of structure protection, visibility enhancement and halo effect, including the structural similarity (SSIM), the percentage of new visible edges, the contrast restoration quality $\bar{r}$ and the fog-aware density evaluator (FADE). According to [11], the indicator SSIM assesses the structural information preserving ability for a dehazing method. The indicators $e$ and rare proposed by [12], in which the indicator $e$ measures the ratio of edges that are newly visible after restoration, and indicator $\bar{r}$ verifies the average visibility enhancement obtained via restoration. The indicator FADE is proposed by [13], which is an assessment of haze removal ability. We compute these four indicators (SSIM, $e \bar{r}$ and FADE) on the dehazing results shown in Figures 3a-3e and the corresponding results listed in Tables 1-4, respectively. In addition, the time consumption comparison within the respective five state-of-the-art methods is shown in Table 5.

Table 1 Comparison of structural similarity (SSIM) for the dehazing results obtained via different methods.

\begin{tabular}{cccc}
\hline SSIM & [7] & {$[16]$} & Ours \\
\hline Figure3a & 0.7238 & 0.6542 & 0.7544 \\
Figure3e & 0.5635 & 0.5284 & 0.5762 \\
\hline
\end{tabular}

Table 2 Comparison of $\mathrm{r}$ for the dehazing results obtained via different methods.

\begin{tabular}{cccc}
\hline $\bar{r}$ & [7] & {$[16]$} & Ours \\
\hline Figure3a & 1.6311 & 4.5631 & 3.5654 \\
Figure3e & 3.7805 & 4.2345 & 5.2764 \\
\hline
\end{tabular}


Table 3 Comparison of e for the dehazing results obtained via different methods.

\begin{tabular}{cccc}
\hline$e$ & {$[7]$} & {$[16]$} & Ours \\
\hline Figure3a & 2.5683 & 2.4607 & 2.5428 \\
Figure3e & 0.3377 & 0.3582 & 0.4173 \\
\hline
\end{tabular}

Table 4 Comparison of fog-aware density evaluator (FADE) for the dehazing results obtained via different methods.

\begin{tabular}{cccc}
\hline FADE & {$[7]$} & {$[16]$} & Ours \\
\hline Figure3a & 0.3984 & 0.4653 & 0.3299 \\
Figure3e & 0.3422 & 0.3943 & 0.2057 \\
\hline
\end{tabular}

Table 5 Comparison of time consumption for the dehazing results obtained via different methods.

\begin{tabular}{cccc}
\hline Time & [7] & {$[16]$} & Ours \\
Consumption & & & \\
\hline Figure3a & 1.46 & 3.23 & 1.12 \\
Figure3e & 3.33 & 2.08 & 0.93 \\
\hline
\end{tabular}

Generally, when value of SSIM is higher, the structural information preserving property for a dehazing method is better. The higher values of e and $r$ indicate better visual improvement after restoration. The lower values of FADE imply less haze residual (which means a better dehazing ability).

The corresponding values of indicator SSIM are listed in Table 1. From Table 1, we can notice that our dehazing results achieve the best SSIM for two hazy images which implies the superior structure information preserving ability of the proposed method. As shown in Table 2, our dehazing results achieve the top values for two hazy images, which verify the robustness and effectiveness of our method for visibility recovering. According to Table 3, our results yield the top value for two images. In contrast, our result removes most of the haze and unveils the majority of the scene objects, which can be further verified via the FADE value as shown in Table 4. The time consumption is another important evaluation factor for a dehazing algorithm. As displayed in Table 5 , the time used to dehaze with our method is shortest in case of preserving quality and efficiency.

\section{Conclusion}

This paper aims at the features of this wearable device. In the hardware platform, firstly, the images are sampled according to the brightness partition. Meanwhile, we calculate the transmittance of images by means of mean filtering. Then, we use guide filter to repair the image. In this way, the white side phenomenon is effectively avoided. At the same time, it solves the problem that the smoke in the fire scene is thicker and the calculation is not refined. Secondly, the dark channel prior theory is combined to calculate the image after fog. The calculation is based on the image degradation model. Finally, we use the straight square equalization algorithm. So the gray uniformity distribution enhances the image contrast. The grayscale will be distributed evenly and the contrast of the image will be enhanced. As the results, the image of the fire scene is clearer. It is effective and fast to realize the fog removal and ensures the quality of the image output of the fire scene. In the process of processing, the method of sampling is introduced to improve the computational efficiency. Due to the estimation of global atmospheric light by mean value, it is effective to avoid overvaluation of atmospheric light. The calculation speed is improved effectively because of no sorting. Based on the results of our study, it can achieve image haze removal in the 
way of real-time, fast and effective, and helps fire fighters conduct site investigation.

\section{Acknowledgements}

This work was supported by Science and Technology Innovation Training Program (No. SZDG2017023), and supported by Smart service system cup national university internet of things technology and application competition (No. 18B519). It is also supported by Jiangsu province key research and development - industry foresight - competition project (No. BE2017111).It also supported by the National Natural Science Foundation of China (No. 61571241) and science and technology project of Nanjing Gulou District (No. 20160205).

\section{References}

[1] Fan Guo, Zixing Cai, Bin Xie and Jin Tang. (2010) Review and Prospect of Image Dehazing. Computer Technology Application, 30 (09), 2417-2421.

[2] Di Wu and Qingsong Zhu. (2015) Recent Advances in the Study of Image Fog. Automation Journal, 41 (02), 221-239.

[3]Xuebao Li, Xusheng Huang, Yanfang Zheng, Yongliang Li, et.al. (2017) Design of Forest Fire Smoke Detection Algorithm Based on Wavelet Transform. Information Technology, 10, 10-13.

[4] Qin Ma. (2017) Application of Image Processing Technology in Ship Fire Smoke Detection System. Ship Science and Technology, 39 (14), 187-189.

[5] Hongchao Tan, Yanyan Li, Wei Long, Ruipeng Zhao and Qian Wang. (2017) Improved Dark Basic Prior Theory Video Dehazing Algorithm Research. Computer Engineering and Application, 10, 1-9.

[6] Weixing Wang, Xiang Xiao and Liangqin Chen. (2015)The Combination of The Minimum Filtering and Guided Filtering of Dark Color to Fog. Optical Precision Engineering, 23 (07), 21002108.

[7] Kaiming He, Jian Sun and Xiaoou Tang. (2010) Single Image Haze Removal Using Dark Channel Prior. IEEE Transactions on Pattern Analysis and Machine Intelligence.

[8] Chao Chen, Xinjue Peng and Lizhuang Ma. (2016) Real-time Adaptive Defog Algorithm of Video. Computer Engineering and Application, 52 (06), 150-155.

[9] Jinbao Wang, Ning He, Lulu Zhang and Ke Lu. (2015) Single Image Dehazing with a Physical Model and Dark Channel Prior. Neurocomputing, 149.

[10] Yanmei Li. (2013) Research on The Related Technology and Application of Image Enhancement. University of Electronic Science and Technology.

[11] Wang, Z., Bovik, A.C., Sheikh, H.R. and Simoncelli, E. P. (2004) Image Quality Assessment: from Error Visibility to Structural Similarity. IEEE Trans. Image Process, 13, 600-612.

[12] Hautière, N., Tarel, J. P., Aubert, D., \& éRIC DUMONT. (2011). Blind Contrast Enhancement Assessment by Gradient Ratioing at Visible Edges. Image Analysis \& Stereology, 27(2), 87-95.

[13] Choi, L. K., You, J., \& Bovik, A. C. (2015). Referenceless Prediction of Perceptual Fog Density and Perceptual Image Defogging. IEEE Transactions on Image Processing, 24(11), 3888901.

[14] The image downloaded from http://safety.gasshow.com/pages/20150212/082006781.html.

[15] The image downloaded from http://roll.sohu.com/20110606/n309410028.shtl.

[16] Kim, T. K., Paik, J. K. and Kang, B. S. (1998) Contrast Enhancement System Using Spatially Adaptive Histogram Equalization with Temporal Filtering. IEEE Transactions on Consumer Electronics, 44(1), 82-87. 